\title{
Hypophosphatemia as Atypical Panic Disorder: A Case Study
}

\author{
Benjamin Kligler, $M D, M P H$
}

Panic disorder is extremely common, affecting 1 to 3 percent of the US population in its fully developed form. ${ }^{1}$ Up to 10 percent of adults experience partial manifestations of panic disorder at some point in their lives. ${ }^{2}$ Frequently the initial symptoms, such as chest pain, dizziness, or paresthesia, are physical, and it can be difficult to decide how aggressively to search for potential biomedical explanations for a symptom once the diagnosis of panic disorder is suspected. In an attempt to balance thorough and conscientious diagnostic testing when intuition suggests the condition is psychogenic, it is not uncommon to do extensive testing before comfortably settling on the diagnosis of panic. This report describes a case of a particularly unusual physical manifestation of panic disorder-hypophosphatemia - and the long road of diagnostic testing traveled before arriving at the appropriate diagnosis and treatment.

\section{Case Report}

A 32-year-old woman with no remarkable medical history first sought treatment at the family practice clinic associated with the Beth Israel Medical Center in December 1995. During the 3 months preceding that visit, she had several episodes of severe weakness with accompanying numbness and tingling in her extremities. On two of these occasions she was seen in a local emergency department, and both times she had abnormally low serum phosphate levels. At the second emergency department visit, her phosphate level was 0.8 $\mathrm{mg} / \mathrm{dL}$ (normal 2.6 to $4.9 \mathrm{mg} / \mathrm{dL}$ ). On that occasion she received phosphate repletion and was referred to an endocrinologist. Her parathyroid hormone, urinary phosphate, and vitamin D levels

Submitted, revised, 2 March 1998.

From the Department of Family Medicine, Beth Israel Medical Center, Albert Einstein College Of Medicine, New York. Address reprint requests to Benjamin Kligler, MD, MPH, Institute for Urban Family Health, 16 East 16th St, New York, NY 10003. were normal. The patient's condition was diagnosed as phosphate depletion, and she was told to continue taking an oral phosphate supplement. She continued to have milder symptoms of fatigue and muscle weakness despite phosphate repletion, and she wanted a second opinion.

Physical examination showed the patient to be a thin woman in no acute distress. Findings during her examination were normal except for a small cyst on the right lower gum and a soft systolic heart murmur. She weighed 109 pounds and reported no recent weight loss. Blood chemistry values at this time were also normal. Her diet was unremarkable for potential phosphate deficiency, and she denied diarrhea, ingestion of any medicinal or herbal products, including antacids, laxatives, or licorice (with the exception of the phosphate supplements), or alcohol use. She could not describe a precipitating factor for any of the episodes and denied any recent major stressors.

The eldest of four siblings, she had been raised in New York. Both parents and all siblings were living, and her family history was unremarkable except for hypothyroidism. She had been married for 6 years, and had been with her husband for 10 years before their marriage. They had two children, aged 2 and 4 years. She had an undergraduate degree in biology and had planned to go into cancer research, but interrupted her studies to raise her family. She described herself as a loner but denied any history of serious anxiety or depression.

She was referred to a consulting endocrinologist at Beth Israel Medical Center, who believed the most likely explanation for the intermittent hypophosphatemia with normal parathyroid hormone, vitamin $\mathrm{D}$, and urinary phosphate levels was oncogenic osteomalacia, a rare condition in which a parathyroid hormone-like humoral factor is secreted by a tumor that could be located anywhere in the body. The typical finding in this disorder is increased urinary phosphate, but because 
the condition can be intermittent, the absence of this finding did not rule out the diagnosis. ${ }^{3}$ The patient was referred to an oral surgeon for excision of the gum lesion, which proved to be a simple inflammatory cyst. During a 1-week trial in which she stopped taking the phosphate supplements, an abnormally low phosphate level could not be documented; however, the patient was also without symptomatic episodes during this period. In January 1996 the results of magnetic resonance imaging (MRI) of her head and neck were negative. A third endocrinologist specializing in phosphate disorders was consulted and agreed that oncogenic osteomalacia was the most likely diagnosis.

From January through May 1996, the patient continued to report episodes of weakness, but none requiring emergency care. Her phosphate levels during this time were normal. At this point those involved in the case began to entertain alternative explanations for her symptoms, including chronic fatigue syndrome, depression, fibromyalgia, and anxiety disorder. An echocardiogram in April 1996 to evaluate her heart murmur was normal. Of note was that the patient described her episodes of weakness as feeling like "she was going to die." She did not want any medication for her condition and declined psychotherapy. Supportive therapy and ongoing phosphate monitoring were continued, and she did, in fact, report diminished symptoms during the subsequent 3 to 4 months.

Beginning in November 1996, she began complaining of worsening symptoms, including pain and paresthesia in the left lower extremity, fullness in the throat, generalized weakness, and "shakiness." Examination findings at this point were remarkable for a weight loss of 8 pounds during the preceding 8 months. Given her normal laboratory values, her condition was continued to be viewed as chronic fatigue syndrome or an anxiety-related disorder, and she was given supportive therapy; however, her weight loss once again raised concerns about an occult malignancy. By February 1997, however, her weight was back to 105 pounds. A consulting neurologist found a positive Lyme titer, for which she received treatment, although it was believed to be an unlikely explanation for her symptoms.

When in May 1997, complaining of profound weakness, she again sought care at a local emergency department, her phosphate level was 0.6 $\mathrm{mg} / \mathrm{dL}$. At this point she had not been taking phosphate supplements for some time. Vitamin D, parathyroid hormone, and urinary phosphate levels were again normal. She was advised to start taking phosphate supplements again, and the search was resumed for an occult malignancy causing oncogenic osteomalacia or for an alternate explanation for her condition. A bone scan was negative. On 3 June 1997 she was seen in the Beth Israel Medical Center Emergency Department for rapid breathing, trembling, and tachycardia. Her phosphate level was $0.6 \mathrm{mg} / \mathrm{dL}$, and she was admitted for further workup.

At admission, an MRI of her chest, abdomen, and pelvis showed normal findings. On two occasions she had staff-witnessed attacks during which appropriate laboratory studies were ordered. At the times of these attacks she complained of weakness and shortness of breath, and she had tachycardia and elevated blood pressures as well as subjective feelings of panic or impending doom. Phosphate levels during both of these episodes were low normal, and on both occasions the medical staff were able to "talk her down" and alleviate her symptoms within 5 to 10 minutes.

At this point an article from the endocrine literature was found that described a rapid and profound drop in phosphate levels in certain patients as a consequence of hyperventilation. ${ }^{4}$ Because nothing was found in the workup for oncogenic osteomalacia, the hypophosphatemia appeared most likely to be a secondary consequence of hyperventilation during a panic episode. Thus the inpatient episodes, which resolved quickly with supportive intervention, did not result in marked hypophosphatemia because the patient did not experience the prolonged hyperventilation she had experienced in the preceding four episodes.

The patient continued to decline medication for her anxiety syndrome. A consulting clinical nurse specialist on the Beth Israel staff who practiced and taught biofeedback and relaxation therapies subsequently saw the patient several times before and then after her discharge and instructed her in techniques of relaxation breathing. During the ensuing 8 months, the patient continued to have occasional mild symptoms of weakness and tremulousness, particularly preceding and during her menses, but she was able to abort her attacks with her breathing and visualization exercises. She had no further emergency visits and required only minimal contact with her physicians. 


\section{Discussion}

This case is instructive because it describes a common problem in the diagnosis of panic disorder: the difficulty of separating a physical condition that is psychogenic from one that is biomedical, such as coronary artery disease or oncogenic osteomalacia. As in many cases where the initial symptom is chest pain, shortness of breath, or palpitations, the biomedical condition to be ruled out could have been life-threatening, which made the situation even more difficult. Hypophosphatemia can cause muscle weakness, irritability or apprehensiveness, and nervous system dysfunction, and it can, in fact, cause death in severe cases. Further complicating the treatment was that the subjective experience of impending doom, which is common and diagnostic in panic disorder, was difficult to attribute to the anxiety disturbance alone when a truly life-threatening condition had not been ruled out.

Because hyperventilation is common with panic disorder, respiratory alkalosis and its accompanying metabolic derangements (including hypophosphatemia) are probably also common in patients who have this disorder. When associated with hyperventilation, hypophosphatemia is caused by an increase in phosphofructokinase activity, an enzyme that represents the rate-limiting step in glycolysis, triggered by a fall in intracellular carbon dioxide levels. Increased phosphorylation triggers increased cellular uptake of phosphate and consequent hypophosphatemia. Had those caring for her remembered the medical axiom, "uncommon manifestations of common disorders occur more frequently than common manifestations of uncommon ones," they might have looked earlier at the possibility of hypophosphatemia secondary to hyperventilation of panic, and some of the extensive diagnostic workup for oncogenic osteomalacia could have been avoided.
As always, looking at the whole patient rather than at the laboratory values in isolation is the best course.

This case is also interesting because the patient's firm refusal to receive any anxiolytic medication despite her profound symptoms forced those caring for her to search for nonpharmaceutical approaches to treatment. Several studies have reported the efficacy of relaxation therapies in treatment of anxiety disorders..$^{5-7}$ In fact, biofeedback and relaxation training succeeded in eliminating the patient's symptoms. Her strong motivation to undertake treatment that did not include medication certainly contributed to this success and suggests that in highly motivated patients, it might be appropriate to attempt a behavioral intervention as first-line rather than secondline therapy.

\section{References}

1. Pary $R$, Lewis S. Identifying and treating patients with panic attacks. Am Fam Physician 1992;46:841-8.

2. Weissman MM. Epidemiology of panic disorder and agoraphobia. Psychiatr Med 1990;8(2):3-13.

3. Ryan EA, Reiss E. Oncogenous osteomalacia. Review of the world literature of 42 cases and report of two new cases. Am J Med 1984;77:501-12.

4. Mostellar ME, Tuttle EP. The effects of alkalosis on plasma concentrations and urinary excretion of inorganic phosphate in man. J Clin Invest 1964;43:138.

5. Benson H, Frankel FH, Apfel R, Daniels MD, Schniewind HE, Nemiah JC, et al. Treatment of anxiety: a comparison of the usefulness of self-hypnosis and a meditational relaxation technique. An overview. Psychother Psychosom 1978;30:229-42.

6. Kabat-Zinn J, Massion AO, Kristeller J, Peterson LG, Fletcher KE, Pbert L, et al. Effectiveness of a meditation-based stress reduction program in the treatment of anxiety disorders. Am J Psychiatry 1992;149:936-43.

7. Raskin M, Bali LR, Peeke HV. Muscle biofeedback and transcendental meditation. A controlled evaluation of efficacy in the treatment of chronic anxiety. Arch Gen Psychiatry 1980;37:93-7. 